\title{
ECMO as a Bridge to Reoperative Cardiac Surgery in a Patient with Cardiogenic Shock and Severe Aortic Insufficiency Due to an Acute Aortic Valve Homograft Failure
}

\author{
Shinya Unai, MD, Vishal Khullar, MD, Haytham Elgharably, MD, Jose L. Navia, MD \\ Department of Thoracic and Cardiovascular Surgery, Cleveland Clinic, Cleveland, Ohio, USA
}

\section{ABSTRACT}

We report a 62-year-old male who had severe aortic insufficiency after a homograft root replacement, requiring venoarterial extracorporeal membrane oxygenation prior to surgery due to profound cardiogenic shock. Severe aortic insufficiency is a contraindication for venoarterial extracorporeal membrane oxygenation, but we were able to stabilize the patient and successfully perform an urgent reoperative surgery.

\section{INTRODUCTION}

Reoperative surgery after a homograft root replacement is challenging. In addition to the usual complications related to a redo-sternotomy, calcifications of the homograft and inflammations of the surrounding tissue make the surgical procedure difficult. We report a patient who presented with profound cardiogenic shock due to ST elevation myocardial infarction (STEMI) and severe aortic insufficiency (AI) 13 years after a homograft root replacement, who underwent a reoperative coronary artery bypass grafting with aortic valve replacement using peripheral veno-arterial extracorporeal membrane oxygenation (VAECMO) as a bridge to surgery. We were able to stabilize the patient with VA-ECMO and successfully perform an urgent reoperative surgery.

\section{CASE REPORT}

A 62-year-old male who underwent an aortic root replacement with a homograft and coronary artery bypass grafting 13 years ago presented with STEMI to an outside facility. He was doing well until the day before admission when he started

Received fanuary 1, 2017; received in revised form fuly 23, 2017; accepted March 7, 2019.

Correspondence: Fose L. Navia, MD, Department of Thoracic and Cardiovascular Surgery, Cleveland Clinic, 9500 Euclid Ave, Cleveland, OH 44195; (216) 444-5104; fax: (216) 445-3272 (e-mail: Naviaf@ccf.org). having chest pain. Coronary angiogram showed occluded grafts, and a percutaneous coronary intervention (PCI) was done to the obtuse marginal branch. Echocardiogram showed severe AI with a dilated left ventricle and an ejection fraction of 20\% (Videos 1(https://vimeo.com/344128524) and 2 (https://vimeo.com/344128696). After the PCI, the patient required high doses of multiple inotrope and vasopressor support, with a rising lactate and creatinine. Peripheral venoarterial ECMO (20Fr femoral arterial cannula, $21 \mathrm{Fr}$ femoral venous cannula, Cardiohelp system (Macquet, Germany) were placed due to hemodynamic instability, as a salvage assist device support. Transcatheter aortic valve replacement (TAVR) was deferred due to concern for infective endocarditis and emergency, thus surgical AVR was planned.

After 24 hours of hemodynamic stabilization, the lactate normalized with a good urine output. The patient was taken to the operating room. He was fully heparinized and the circuit was switched to cardiopulmonary bypass prior to opening the sternum. The ascending aorta was cannulated and the outflow was switched from femoral artery, and SVC was cannulated for drainage. Antegrade and retrograde blood cardioplegia were administered to maintain cardiac arrest. We opened the ascending aorta, and the calcified wall of the homograft was removed. The 3 leaflets of the homograft were completely destroyed due to degeneration (Figure). There was no evidence of infection. The valve was replaced with a \#23 CE valve and the aorta was reconstructed using a bovine pericardial patch. In addition, coronary artery bypass grafting was done using a reverse saphenous vein graft to the LAD and acute marginal of the RCA with 7-0 Prolene. Proximal anastomosis of the vein grafts were done to the bovine pericardial patch using a 5-0 running Prolene suture. The heart was deaired and the cross clamp was removed. Intraoperative TEE showed an LVEF of $40 \%$ with moderate RV dysfunction on mild inotropic support. An intraaortic balloon pump was placed, the ECMO cannulas from the groin were removed, and protamine was given. The chest was left open with a vacuum dressing due to severe coagulopathy. Chest closure was done on postoperative day (POD) 3. The patient was extubated on POD 8. Postoperative echocardiogram showed a well functioning valve with no regurgitation and good biventricular function (Video 3 https://vimeo.com/345681460). He was discharged to a rehabilitation facility on POD 26. 


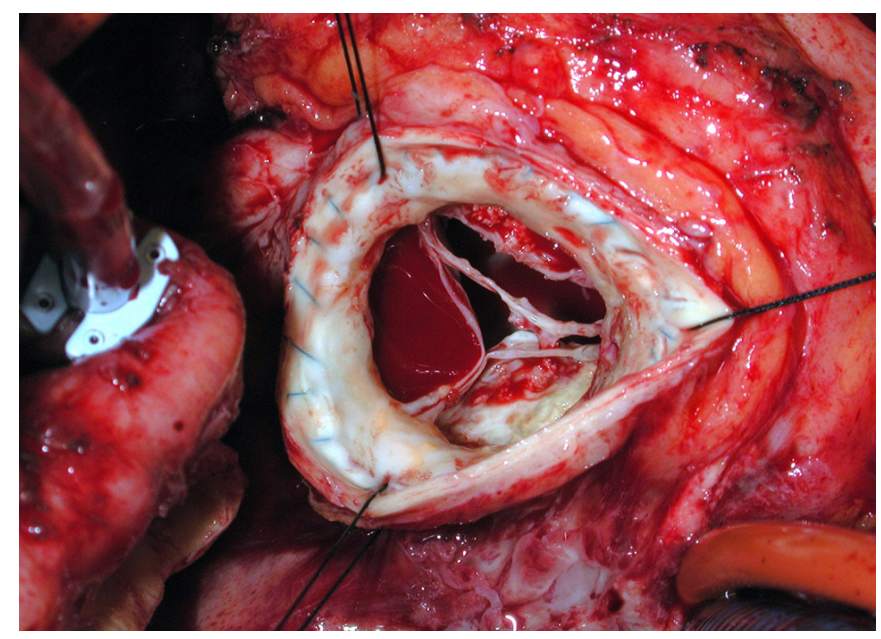

Intraoperative image of the aortic valve.

\section{DISCUSSION}

Homograft replacement of the aortic root is reported to have excellent hemodynamics; however, the long-term durability does not seem to be superior to biological valve prosthesis [O'Brien 2001; Kilian 2010]. The degeneration of the homograft, leading to fibrosis and calcification of the annulus and the aortic wall, make it challenging when a reoperation is necessary. Aortic valve replacement may not be feasible, and explanting the homograft and replacing the root may be necessary, which is reported to have a high rate of mortality [Kowert 2012]. Transcatheter valve replacement is an alternative approach [Dainese 2010], but in our case there was a concern of endocarditis, so surgical approach was selected. Due to poor cardiac function and the critical condition requiring ECMO, rather than replacing the entire root, we were able to replace the valve, remove the calcified aortic wall of the homograft, and perform an aortoplasty with a bovine pericardium patch.
$\mathrm{AI}$ is regarded as a contraindication for ECMO support. The high afterload from the retrograde flow can worsen the degree of $\mathrm{AI}$ and can cause $\mathrm{LV}$ distention and a decrease in the chance of myocardial recovery. However, in our case, with rapidly deteriorating hemodynamics in a patient with prior sternotomy, we had no option left other than placing the patient on ECMO with a backup plan of transvenous placement of an $L V$ vent via a balloon atrial septostomy in case of $L V$ dilatation and low blood flows. This was not necessary, and the ECMO support provided hemodynamic stability and adequate flows to achieve end-organ recovery; also, this preoperative strategy gave us time to perform necessary preoperative evaluation and surgical planning to safely accomplish a high-risk reoperation. The key to prevent LV dilatation is to maintain pulsatility with inotropic support and to avoid unnecessary high flows, which will increase afterload resulting in $\mathrm{LV}$ distension. In cases of complete standstill of the $\mathrm{LV}$ with severe aortic regurgitation, our approach will likely not be successful and would require a more aggressive approach to decompress the LV.

Our case demonstrates the benefit of using ECMO as a salvage device to maintain systemic circulation in a patient who has hemodynamic collapse due to acute aortic insufficiency. Although aortic insufficiency is a contraindication for ECMO, we were able to achieve hemodynamic stability until definitive surgical treatment, which resulted in a good outcome.

\section{REFERENCES}

Dainese L, Fusari M, Trabattoni P, et al. 2010. Redo in aortic homograft replacement: transcatheter aortic valve as a valid alternative to surgical replacement. J Thorac Cardiovasc Surg 139:1656-7.

Kilian E, Fries F, Kowert A, et al. 2010. Homograft implantation for aortic valve replacement since 15 years: results and follow-up. Heart Surg Forum 13:E238-42.

Kowert A, Vogt F, Beiras-Fernandez A, et al. 2012. Outcome after homograft redo operation in aortic position. Eur J Cardiothorac Surg 41:404-8.

O'Brien MF, Harrocks S, Stafford EG, et al. 2001. The homograft aortic valve: a 29 -year, $99.3 \%$ follow up of 1,022 valve replacements. J Heart Valve Dis 10:334-44; discussion 335. 